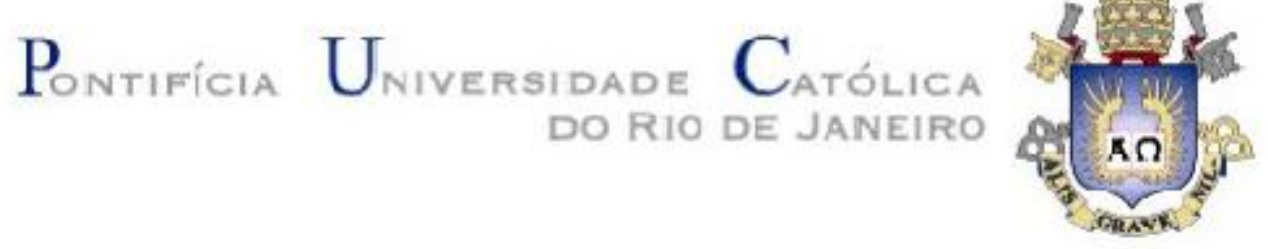

Fábio Luiz Rodrigues

\title{
O primeiro silicone a gente nunca esquece: do implante de prótese mamária em meninas adolescentes
}

Dissertação de Mestrado

Dissertação apresenta como requisito parcial para obtenção do grau de Mestre pelo Programa de Pós-graduação em Psicologia do Departamento de Psicologia da PUC-Rio.

Orientadora: Profa. Junia de Vilhena 


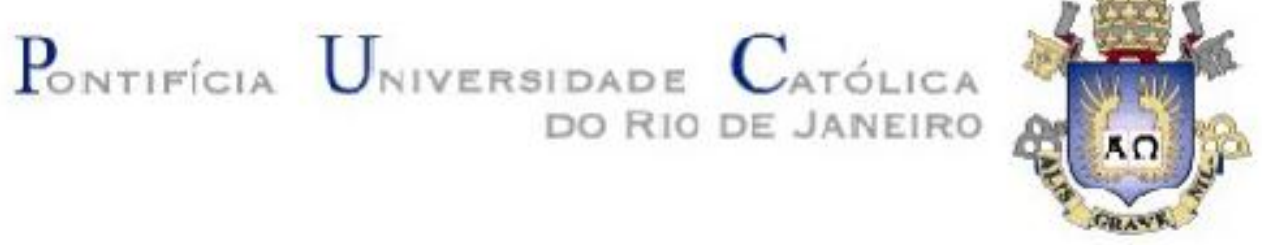

Fábio Luiz Rodrigues

\section{O primeiro silicone a gente nunca esquece: do implante de prótese mamária em meninas adolescentes}

Dissertação apresentada como requisito parcial para obtenção do grau de Mestre pelo programa de Pós-Graduação em Psicologia Clínica do Departamento de Psicologia do Centro de Teologia e Ciências Humanas da PUC-Rio. Aprovada pela Comissão Organizadora abaixo assinada.

Profa. Junia de Vilhena

Orientadora Departamento de Psicologia - PUC-Rio

Profa. Joana de Vilhena Novaes LIPIS/ V.R.C./ PUC-Rio

Profa. Nadja Barbosa Pinheiro Departamento de Psicologia - UFPR

Profa. Denise Berruezo Portinari Coordenadora Setorial de Pós-Graduação

e Pesquisa do Centro de Teologia e Ciências Humanas - PUC-Rio

Rio de Janeiro, 09 de março de 2012 
Todos os direitos reservados. É proibida a reprodução total ou parcial do trabalho sem a autorização da universidade, do autor e da orientadora.

Fábio Luiz Rodrigues

Graduou-se em Psicologia na UTP - Universidade Tuiuti do Paraná em 1996.

Ficha Catalográfica

Rodrigues, Fábio Luiz

O primeiro silicone a gente nunca esquece: do implante de prótese mamária em meninas adolescentes / Fábio Luiz Rodrigues ; orientadora: Junia de Vilhena. 2012.

$133 \mathrm{f.} ; 30 \mathrm{~cm}$

Dissertação (mestrado) - Pontifícia Universidade Católica do Rio de Janeiro, Departamento de Psicologia, 2012.

Inclui bibliografia

1. Psicologia - Teses. 2. Cirurgia estética. 3. Prótese mamária de silicone. 4. Corpo. 5. Adolescência. 6. Psicanálise. 7. Cultura. I. Vilhena, Junia de. II. Pontifícia Universidade Católica do Rio de Janeiro. Departamento de Psicologia. III. Título.

CDD: 150 
Para Décio, companheiro na estrada que, junto com Isabel, estendeu-me a mão, possibilitando-me ancorá-la

na terceira margem do Rio 


\section{Agradecimentos}

A Junia de Vilhena, que subversivamente na academia, pode me (des)orientar ao me conceder tempo - convocando-me diante das minhas páginas em branco -, um tempo além, mas dentro dos dois anos necessários para a escritura de uma dissertação de mestrado.

Aos professores do Programa de Pós-Graduação da PUC-Rio, em especial Marcus André Vieira e Ana Maria Rudge.

A professora Ieda Tucherman, da ECO - UFRJ pelo acolhimento em sua disciplina "Comunicação e Imaginário Tecnológico" e disponibilidade em contribuir com a feitura deste trabalho.

Ao meu grupo de pesquisa, em especial Deborah e Marina, e aos paraenses do PROCAD, Igor, Amanda e Alex.

A Joana de Vilhena Novaes, cuja produção acadêmica acompanho desde 2008, pela afinidade do tema de pesquisa e disponibilidade em contribuir com suas ideias.

A Nadja Pinheiro, pela disponibilidade de participar da banca que avaliou esse trabalho.

A Paulo Becker, da Escola Letra Freudiana, pelo ouvido amigo.

A Dr ${ }^{\mathrm{a}}$ Ruth Graf, cirurgiã plástica que acolheu o trabalho da psicologia em sua clínica, como poucos podem acolher, fomentando assim meu retorno à academia.

A minha família, pelo sempre apoio. Aos meus avós Aldmaro e Therezinha Silveira (in memorian) que me apresentaram, com suas histórias, a cidade do Rio de Janeiro.

A Marcelina, pela prontidão e ajuda.

A CAPES e à PUC-Rio pelos auxílios concedidos. 


\section{Resumo}

Rodrigues, Fábio Luiz; Vilhena, Junia de (Orientadora). O primeiro silicone a gente nunca esquece: do implante de prótese mamária em meninas adolescentes. Rio de Janeiro, 2012. 133p. Dissertação de Mestrado - Departamento de Psicologia - Pontifícia Universidade Católica do Rio de Janeiro.

O implante de prótese mamária de silicone a que meninas adolescentes se submetem na atualidade se tornou um fenômeno cultural sem precedentes. Baseado em tal fenômeno, este trabalho pretende investigar o que move meninas adolescentes a se submeterem ao referido procedimento cirúrgico, a partir da noção de corpo representado para a psicanálise em articulação com o campo da cultura. $\mathrm{O}$ campo de pesquisa foi estabelecido a partir de declarações de meninas adolescentes que se submeteram ou iriam se submeter ao implante de prótese mamária de silicone, coletadas na mídia, através da internet com seus blogs, redes sociais e artigos jornalísticos. As considerações finais deste trabalho ressaltam que, diante da chamada crise da adolescência, o implante de prótese mamária de silicone a que meninas adolescentes se submetem remete ao projeto de alcançar um padrão de beleza imaginário que funcionaria como suporte subjetivo para tal crise.

\section{Palavras-chave}

Cirurgia estética; prótese mamária de silicone; corpo; adolescência; psicanálise; cultura. 


\section{Abstract}

Rodrigues, Fábio Luiz; Vilhena, Junia de (Advisor). The first silicone we never forget: of breast implants in adolescent girls. Rio de Janeiro, 2012. 133p. MSc. Dissertation - Departamento de Psicologia - Pontifícia Universidade Católica do Rio de Janeiro.

The silicone breast implants that adolescent girls undergo nowadays have become an unprecedented cultural phenomenon. Based on this phenomenon, it is intended to investigate what makes adolescent girls undergo such procedure from the idea of body representation for psychoanalysis interrelated with the idea of body for medicine and the field of culture. The research field was based on statements of adolescent girls who had or would have the silicone breast implants surgery, as well as media search, through the internet, with its blogs, social networks and journalistic articles. Results highlight that, facing with the so called adolescent crisis, the silicone breast implants which adolescent girls undergo take them to the project of reaching an imaginary standard of beauty that would work as a subjective support for such crisis.

\section{Keywords}

Plastic surgery; silicone breast implants; body, adolescence; psychoanalysis; culture. 


\section{Sumário}

1. Introdução

2. O Corpo na cultura 17

2.1 O primeiro Valisère - Um recorte do corpo na cultura 17

2.2 O corpo feminino em voga na contemporaneidade 19

2.3 Cirurgia estética, uma técnica corporal 23

2.4 A revelação da beleza feminina na história 25

2.5 Da beleza à felicidade 31

2.6 O corpo fabricado, o dispositivo tecnológico 35

3. O Corpo para a psicanálise 40

3.1 O percurso do capítulo 40

3.20 corpo histérico (representado) x O corpo orgânico 43

$3.3 \mathrm{O}$ recalque (ou a visível invisibilidade do corpo) 46

3.4 O complexo de Édipo na menina 49

$3.5 \mathrm{O}$ declínio do conflito psíquico para Ehrenberg 52

3.6 Narcisismo e adolescência $\quad 59$

3.7 "Isso de querer ser exatamente o que se é ainda vai nos levar além" 66

3.8 Feios, Perfeitos, Especiais $\quad 69$

4. Campo 76

4.1 Descrevendo o campo 76

4.1.1 A chegada 76

4.1.2 Tentativas e impasses $\quad 76$

4.1.3 Aspectos metodológicos $\quad 78$

4.1.4 A (quase) invisibilidade de uma prótese de silicone... 81

4.2. Categorias de análise 86

4.2.1 Corpos indecisos: "turbinar" ou não "turbinar"?

$\begin{array}{ll}\text { Eis a questão } & 87\end{array}$

4.2.2 Corpos presenteados: "hoje faço 15 anos!" 89 
4.2.3 Corpos midiatizados: "ou você tem beleza, tem peitão ou não é ninguém" 91

4.2.4 Corpos advertidos: "seios realmente insuficientes?" 94

4.2.5 Corpos em transformação: "a adolescência desperta no horizonte" 98

4.2.6 Corpos fusionados: "tal mãe, tal filha" 102

4.2.7 Corpos simetrizados: "o ideal do tamanho" 108

4.2.8 Corpos tímidos: “a vergonha é maior que o desejo!!” 111

4.2.9 Corpos desejados: "o olhar masculino" 114

4.2.10 Corpos em competição: "little miss sunshine e miss teen brasil"

5. Considerações finais

6. Referências bibliográficas 
Enquanto leio meus seios estão a descoberto É difícil concentrar-me ao ver seus bicos Então rabisco as folhas deste álbum Poética quebrada pelo meio

Enquanto leio meus textos se fazem descobertos É difícil escondê-los no meio dessas letras Então me nutro das tetas dos poetas pensados no meu seio 